\title{
Wenn das liebe Geld nicht wär': Zur Konstruktion von Intimität zwischen Tragemüttern und gleichgeschlechtlichen Männerpaaren ${ }^{1}$
}

Die wachsenden Möglichkeiten der Reproduktionsmedizin stellen alltagsweltlich unhinterfragte Gewissheiten fundamental zur Disposition: Wie gründen Menschen eine Familie? Was ist überhaupt eine Familie? Und wie viele Personen sind an der Entstehung eines Menschen beteiligt? Heteronormative und essentialistische Konzeptionen von Reproduktion, Fortpflanzung, Verwandtschaft, Familie und Sexualität verlieren an Eindeutigkeit: Reproduktionsmedizinische Methoden wie Eizell- oder Samenspende, In-vitro-Fertilisation oder Tragemutterschaft bieten unterschiedlichen Menschen, ob lesbisch, schwul, bi, hetero, trans` oder queer, vielfältige Möglichkeiten ihren Kinderwunsch zu realisieren.

Dabei werden insbesondere Tragemutterschaft und Eizellspende in Bezug auf rechtliche Regulierungen, medizinische Risiken, bioethische und kapitalismuskritische Positionen zur Inwertsetzung des Körpers (z. B. Lettow 2011) in Deutschland und international kontrovers diskutiert (Cooper/Waldby 2014). Dem zugrunde liegt der scheinbar unüberwindbare Widerspruch zwischen zwei zentral im feministischen Denken und Handeln verankerten Positionen: Die körperliche und reproduktive Selbstbestimmung von Frauen auf der einen (z.B. Andrews 1995; Shultz 1990) sowie die Kommodifizierung und Ausbeutung weiblicher Reproduktionsarbeit auf der anderen Seite (z. B. Corea 1985; Raymond 1993). Denn bei einer kommerziellen Tragemutterschaft wird eine Frau $^{2}$ dafür bezahlt,

1 Für kritisches Feedback und Unterstützung im Überarbeitungsprozess danke ich Aline Oloff, den Herausgeberinnen Friederike Kuster und Katharina Liebsch, den beiden für mich anonym geblieben Gutachter_innen sowie meinen Kolleg_innen Almut Peukert, Lilian Hümmler, Leoni Linek, Loui Schlecht und Julia Bringmann.

2 Wenn ich im Folgenden von »Frauen« als Tragemütter und Eizellproduzentinnen spreche, dann ist dies auch der cisnormativen und binären Zweigeschlechterlogik des Forschungsfeldes Reproduktionsindustrie geschuldet. Zum einen zeigen meine Beobachtungen in diesem Feld, dass Reproduktionskliniken und Eizellagenturen davon ausgehen, dass eizellspendende Personen sowie Personen, die das Kind anderer Eltern (gegen Geld) gebären, sich ausschließlich cisgeschlechtlich als Frauen (und hetero- 
dass sie schwanger wird, um ein Kind für ein Paar oder Single auszutragen. Gleichgeschlechtliche männliche Paare oder Personen ohne eigene Eizellen benötigen darüber hinaus die Hilfe einer weiteren Person, die ihre Eizellen (oft gegen hohe Aufwandsentschädigung) >spendet.. Die Eizellen werden dann mit dem Sperma (z. B. der werdenden Väter) befruchtet und in den Uterus einer Tragemutter implantiert. Beide Personen - Tragemutter wie Eizellproduzentin - geben ihre elterlichen Rechte an dem Kind entweder vor oder nach der Geburt an die intendierten Eltern ab.

Ausgangspunkt für diesen Artikel ist die komplexe Verwobenheit von Geschlechterverhältnissen, Sexualität, Reproduktion(sarbeit), Intimität und Ökonomie. Der Beitrag behandelt das Phänomen der Tragemutterschaft bei gleichgeschlechtlichen Cis-Männerpaaren, da sich hier ein besonderes Spannungsverhältnis zeigt: zwischen der Kommodifizierung weiblicher Reproduktionsarbeit wie Eizellgabe, Schwangerschaft und Geburt sowie den Möglichkeiten für schwule Paare, eine Elternschaft in einer von ihnen präferierten Familienkonstellation mit Hilfe von Reproduktionstechnologien umzusetzen. Mit Shellee Colens (1995) Konzept der "stratified reproduction" geraten die strukturellen Ungleichheiten und Machtverhältnisse zwischen den Personen, die ihre reproduktive Arbeit anbieten und jenen, die sie in Anspruch nehmen, in den Blick. Welche Menschen Zugang zu Reproduktionsmedizin haben, hängt mit deren ökonomischen Ressourcen, rechtlichen Anerkennungs- sowie Teilhabechancen zusammen. Werden die reproduktiven Entscheidungen von heterosexuellen, verheirateten und gut verdienenden Paaren eher unterstützt, werden andere behindert oder abgehalten. Schwule Paare verletzen im Kontext der Familiengründung durch Tragemutterschaft (mindestens) zwei gesellschaftliche Normen: Die Freiheit der Privatsphäre von Vermarktlichung sowie heteronormative Vorstellungen von Elternschaft (Smietana 2017).

Dieses Spannungsverhältnis diskutiere ich im Folgenden auf der Grundlage teilnarrativer Interviews, die ich mit gleichgeschlechtlichen männlichen Paaren aus Israel und Deutschland geführt habe, die mit Hilfe kommerzieller Tragemutterschaft in den USA ihren Kinderwunsch ver-

sexuell) identifizieren (vgl. dazu auch das Interview »I'm a Queer Egg Donor« auf dem Blog http://www.weareeggdonors.com vom 13.11.2015). Zum anderen wird die reproduktive Arbeit vor allem von Cis-Frauen ausgeführt und die Vergeschlechtlichung ihrer reproduktiven Arbeit möchte ich sichtbar machen. Trans` Personen werden - soweit ich das Feld und den Forschungsstand überblicke - i.d.R. als Rezipient_innen der Fertilitätsindustrie gerahmt, denen "fertility options" zur Verfügung gestellt werden (z.B. Kryokonservierung von Ei- und Samenzellen). Im Kontext der Fortpflanzungsindustrie hält sich die Vorstellung einer vermeintlich natürlichen Zweigeschlechtlichkeit besonders hartnäckig. 
wirklicht haben. Dabei konzentriere ich mich auf die Verstrickung der als konträr und unvereinbar gerahmten Bereiche des Intimen und des Wirtschaftens in familialen Praktiken. Während in der bürgerlichen Kleinfamilie der Widerspruch zwischen Geld und Liebe »institutionell gezähmt» (Wimbauer 2003, 129 ff.) ist, ${ }^{3}$ scheint die Bedeutung von Geld in Tragemutterschaftsbeziehungen besonders begründungsbedürftig, weil neben dem Geld noch das als "falsch" gelesene, nicht-heterosexuelle Elternpersonal hinzukommt.

Anhand der Rekonstruktion des empirischen Materials entfalte ich das Argument, dass die Paare ein Narrativ über ihre Beziehung zur Tragemutter entwickeln, in dem die affektive und intime Beziehung dominiert, während die ökonomische Ebene verschleiert werden muss. Ich zeige, wie die Paare dafür bekannte soziale Praktiken rund um Schwangerschaft nutzen. Sie konstruieren die Schwangerschaft als gemeinsame Erfahrung, um sich retrospektiv darauf positiv beziehen können. Zwar lebt die schwangere Frau - meist mit ihrer eigenen Familie - in den USA, doch die Paare nehmen über große Entfernungen hinweg im Rahmen ihrer Möglichkeiten aktiv an der Schwangerschaft teil und unterstützen die Tragemutter. Die Tragemutter hat wiederum die Rolle der Vermittlerin zwischen den intendierten Eltern und ihrem zukünftigen Kind.

Im ersten Teil konzentriere ich mich zunächst auf die Beziehungswerdung: Wie wird diese in den Narrativen dargestellt und welche Praktiken lassen sich daraus rekonstruieren? Darauf aufbauend analysiere ich die komplexen Verstrickungen zwischen Marktförmigkeit und familialer Intimität in Tragemutterschaftsbeziehungen anhand von Schwangerschaftserzählungen.

\section{Empirische Studie: Schwule Paare in Tragemutterschaftsarrangements}

Die empirische Grundlage dieses Artikels sind 26 teil-narrative Interviews mit 15 Cis-Männerpaaren aus Israel und Deutschland, die sich für eine Tragemutterschaft in den USA entschieden haben. ${ }^{4}$ Ein Vergleich der beiden

3 Das Ideal der romantischen Liebe sowie die geschlechterbinäre Vorstellung einer Rollenkomplementarität verschleiern die institutionell legitimierte Abhängigkeit der Ehefrau von ihrem Ehemann, die sich aus dem - vom Alleinernährer verdienten - Familieneinkommen ergibt (Wimbauer 2003).

4 Die Forschung wurde von der Deutschen Forschungsgemeinschaft (DFG) im Rahmen eines Promotionsstipendiums in der International Research Training Group 
Länder bot sich aufgrund der unterschiedlichen rechtlichen Regulierungen zu Reproduktionstechnologien an: In Deutschland sind Tragemutterschaft und Eizellspende verboten, in Israel erlaubt. Die Interviewpartner_innen sind zwischen 29 und 53 Jahre alt. Alle bis auf ein Paar sind in einer Lebenspartnerschaft bzw. haben im Ausland geheiratet. Sie sind wirtschaftlich gut situiert, haben ein abgeschlossenes Studium und mindestens einer der Partner arbeitet in einer gehobenen Position wie Geschäftsführung oder höherer Dienst. Paare, die bereits Kinder haben, habe ich einmal interviewt, während ich mit den Paaren, die ich im Prozess ihrer Elternwerdung traf, mindestens zweimal bis viermal über den Verlauf gesprochen habe. Darüber hinaus habe ich acht Wochen ethnographisch in zwei Surrogacy Agencies und einer Fertilitätsklinik in den USA geforscht und Expert_inneninterviews mit Ärzt_innen, medizinischem und labortechnischem Personal, Anwält_innen, Fallmanager_innen und Tragemüttern geführt.

Dem interpretativen Paradigma folgend habe ich mich bei der Auswertung auf die subjektiven Deutungen und Interpretation der Interviewten konzentriert. Im Sinne der Grounded Theory (Corbin/Strauss 2008) wurden zentrale Segmente der Interviews analysiert sowie die empirischen Ergebnisse mit Kolleg_innen diskutiert, um Qualität, Transparenz und die intersubjektive Nachvollziehbarkeit der Analyse sicherzustellen.

In diesem Artikel kommen Paare zu Wort, die entweder schon Kinder hatten oder für die eine Tragemutter mit ihrem Kind schwanger war. Es handelt sich um exemplarische Fälle, an denen das interaktive Handeln zur Herstellung sozialer Ordnung im Kontext von Tragemutterschaftsarrangements analysiert und rekonstruiert wurde. Die Erzählungen sind eine retrospektive Sicht der Paare auf die Ereignisse und Ausdruck ihrer subjektiven Sinndeutungen. Die Darstellungen ihrer Erlebnisse und Erfahrungen wurden in den Interviews situativ produziert und das Interview damit performativ als "soziale Situation mit einer eigenen Wirklichkeit hergestellt« (Deppermann 2013, 46).

\section{Beziehungswerdung im Spannungsfeld von Markt und Intimität}

Tragemutterschaft findet in einem Feld statt, in dem Technologie, Marktinteressen, Recht, unterschiedliche ökonomische Ressourcen sowie gesellschaftlich institutionalisierte Normen von Sexualität, Verwandtschaft und

'Human Rights under Pressure - Ethics, Law and Politics` an der Freien Universität Berlin (2014-2017) gefördert. 
Familie aufeinandertreffen. Frauen bieten als reproduktive Arbeiterinnen ihre körperliche Leistungsfähigkeit an, die durch die zur Verfügung stehenden Technologien verwertbar gemacht und mit dem Mehrwert aus Gametenentnahme, In-vitro-Fertilisation und dem weiblichen Reproduktionsvermögen produziert wird (Moreno 2016, 238). Gleichzeitig werden in diesem ökonomisierten Kontext unterschiedliche körperliche wie emotionale Beziehungen geschaffen: Zwischen den Tragemüttern und den intendierten Eltern, zu Eizellproduzentinnen, zum medizinischen Fachpersonal in der Fertilitätsklinik, zu den Angestellten der Tragemutterschaftsagenturen oder Anwält_innen. Den Beziehungen liegt der Tausch von Gameten, medizinischem oder rechtlichem Expert_innenwissen, von Fürsorgearbeit oder der Leihgabe reproduktiver Organe wie der Gebärmutter, gegen ein Entgelt zugrunde. Diese Verknüpfung von materiell-ökonomischen mit sozialen Beziehungen und den damit einhergehenden Machtverhältnissen löst in öffentlichen Diskursen häufig großes Unbehagen aus. ${ }^{5}$

Denn Tragemutterschaft fällt in den Bereich der intimen Industrien (Parreñas et al. 2016): Intime Arbeit, die vermeintlich keinen Preis hat, wird hier gegen Geld statt gegen Liebe getauscht. Auftraggebende Eltern müssen zur Umsetzung ihres Kinderwunsches viel Geld investieren. Dies trifft auch auf konventionelle Liebes- und Verwandtschaftsbeziehungen zu. Dort werden die ökonomischen Aspekte mit dem Narrativ der "pure relationship" (Giddens 1992) jedoch verdeckt: Während es in der (heterosexuellen) Familie eine institutionalisierte De-Thematisierung von Geld und Liebe gibt (Wimbauer 2003), muss diese Kopplung durch soziale Interaktionen in Tragemutterschaftsbeziehungen im Kontext des Privaten und Intimen wie Schwangerschaft und Elternwerden erst noch unsichtbar gemacht werden.

Ich rekonstruiere dies im Folgenden exemplarisch und ausführlich anhand des Paares Ingo und Dieter Droste und ihrer Beziehungswerdung mit der Tragemutter Despoina. Der Fokus liegt auf Ereignissen, denen sie besondere Bedeutung zuschreiben, um ihre persönliche Beziehung zu Despoina darzustellen.

Das Paar Ingo und Dieter Droste besuche ich in ihrem Zuhause in der süddeutschen Provinz. Hier leben die beiden mit ihren drei Kindern: Ihr erster Sohn Diego geht schon in den Kindergarten und seit ein paar Monaten sind sie Eltern von Zwillingen. Ingo arbeitet in der Geschäftsfüh-

5 Dies gilt insbesondere für die Entlohnung von Schwangerschaft. Schwangerschaft und Mutterschaft sind höchst vergeschlechtlichte soziale Praktiken, die im Kontext von Tragemutterschaft oft biologisch essentialisierend diskutiert werden (Teman 2010). 
rung eines Autoherstellers, während Dieter vor der Geburt der Kinder als Angestellter beschäftigt war und zum Interviewzeitpunkt als Hausmann die Sorgearbeit übernimmt. Die beiden sind seit 25 Jahren ein Paar. Vor zehn Jahren haben sie gemeinsam das Haus gebaut, in dem sie jetzt wohnen und zwei Jahre später eine Lebenspartnerschaft geschlossen. In dieser Zeit begann vor allem Ingo über ein gemeinsames Kind nachzudenken.

Der Entscheidung, eine Familie mit Kindern mithilfe einer Tragemutter zu gründen, geht meistens ein umfänglicher Prozess des Abwägens unterschiedlicher Möglichkeiten der Elternwerdung wie Adoption, Co-Parenting oder Pflegschaft voraus. Für die von mir interviewten Paare war Adoption keine realistische Möglichkeit, weil sie zum Zeitpunkt der Interviews gleichgeschlechtlichen Paaren in Deutschland nicht offenstand. In Israel können gleichgeschlechtliche Paare seit 2008 gemeinsam ein Kind adoptieren, aber ihre Chancen sind sehr gering. ${ }^{6}$ Adoption geht aus Sicht einiger Paare mit vielen Ungewissheiten einher. Eine Tragemutterschaft verspricht demgegenüber Planungssicherheit und bietet im Unterschied zum Co-Parenting die Möglichkeit einer Zweielternfamilie. Auch die genetische Verwandtschaft zwischen dem Kind und einem der Väter ist für viele der Paare entscheidend.

Diese unterschiedlichen Optionen hat auch Ingo abgewogen. Er knüpfte schließlich im Internet Kontakt zu einem Paar, mit dem er sich über die Möglichkeiten der Tragemutterschaft austauschte. Sie empfahlen ihm eine Tragemutterschaftsagentur in den USA. Kurz nach dem ersten Kontakt mit der Agentur stellte ihnen ihre Fallmanagerin eine potentielle Tragemutter vor - Despoina. Wenige Monate später reisten Dieter und Ingo unter dem Vorwand eines Winterurlaubs in die USA. Sie mussten sich diversen medizinischen Tests unterziehen, eine Samenspende abgeben und lernten Despoina persönlich kennen. Ingo erinnert sich, dass ihr erstes Treffen in einem Café stattfand. Im Anschluss an dieses erste Kennenlernen lud Despoina die beiden ein, gemeinsam Thanksgiving mit ihrer Familie und ihren Nachbar_innen zu feiern. Ingo erzählt: „Und dann waren wir bei ihr, haben dann Thanksgiving da noch gefeiert und so war das ganz nett bei Nachbarn. Und da ham wa gleich gemerkt, das ist super. Das funktioniert. Also wir waren uns auch sympathisch so.«(Int. 1, Paar Droste)

Als Gäste bei einem Familienfest können Ingo und Dieter nicht nur Despoina, sondern auch ihre Familie sowie ihre Nachbar_innen in einem privaten Rahmen kennenlernen. Die Tragemutter im Umfeld ihrer sozia-

6 Zwischen 2008 und 2017 haben in Israel insgesamt nur drei gleichgeschlechtliche Paare ein Kind adoptiert (Yaron 2017). 
len Nahbeziehungen und außerhalb des Agenturkontextes zu erleben, wird auch in anderen Interviews von den Paaren als äußerst erleichternd empfunden, da in diesen Situationen die Regulierung und Strukturierung ihrer Interaktionen durch die Fallmanager_innen der Agentur entfällt. Ingo hat positive Erinnerungen an das Treffen und er nimmt eine erste Bewertung der Beziehung vor, die zum einen den technisch-funktionalen Charakter zum Ausdruck bringt und zum anderen die gegenseitige Sympathie betont. Er bringt eine spontane Verbundenheit sowie eine gefühlsmäßige Übereinstimmung zum Ausdruck, was zentral für den Aufbau einer langfristigen und über die Schwangerschaft hinausgehenden Beziehung ist. Die langfristige Beziehung ist (auch) deshalb so wichtig, weil sie als gleichgeschlechtliche Eltern im Unterschied zu nicht gleichgeschlechtlichen Eltern nicht vertuschen können, dass sie die Hilfe von mindestens zwei Frauen (Eizellproduzentin und Tragemutter) in Anspruch genommen haben, um ihren Kinderwunsch zu verwirklichen. So wollen sie ihren Kindern eine transparente Geschichte erzählen, wie diese entstanden und auf die Welt gekommen sind. Zudem müssen Ingo und Dieter als werdende Eltern beweisen, dass sie verantwortungsvolle und selbstreflexive Entscheidungen treffen. Zwar gibt es die Beziehung zu Despoina nur, weil sie eine Agentur dafür bezahlt haben, diese Tragemutterschaftsbeziehung zu arrangieren. Allerdings steht die Bedeutung des guten Verhältnisses und der gegenseitigen Zuneigung im Zentrum, während das Finanzielle einem Latenzschutz unterliegt und außen vorgelassen wird. ${ }^{7}$ Dies wird gestützt durch die Agenturen, die fast ausschließlich Bilder und Narrative von familialer Zuneigung, Liebe oder dem Geschenk der Elternschaft aufrufen - sei es im Infomaterial, auf ihren Webseiten oder im persönlichen Gespräch.

In den paarinternen Aushandlungen setzen sich viele Paare auch mit moralischen und ethischen Fragen zu Tragemutterschaft sowie deren Marktförmigkeit auseinander. Rudrappa und Collins (2015, 942) argumentieren für den indischen Kontext, dass gerade weil Tragemutterschaft als "morally suspect activity" gilt, intendierte Eltern Narrative der Verbundenheit und Zuneigung entwickeln müssen. Für eine soziologische Analyse dieser Praktiken bietet sich Ken Plummers Konzept (2003) der grounded everyday moralities an, um zu rekonstruieren, wie Akteur_innen in ihren Alltagspraxen mit moralischen Dilemmata umgehen, die aus technologischem Fortschritt, Globalisierung und komplexer werdenden For-

7 Meistens vermittelt die Agentur bei offenen Fragen und Schwierigkeiten zwischen den Paaren und der Frau. Vertragliche oder finanzielle Belange werden selten von den Dreien direkt besprochen, sondern von und über die Fallmanager_innen geregelt, damit die Beziehung zwischen ihnen störungsfrei bleiben kann. 
men von Intimität entstehen. Faircloth und Gürtin (2018) fassen dies als Reflexivität in den reproduktiven Entscheidungen: Normative und moralische Erwartungen an Reproduktion müssen von den Eltern antizipiert und entsprechend in ihr reproduktives Handeln übersetzt werden.

Vivanna Zelizer (2005) folgend, kommt es bei der Überschneidung der vermeintlich separaten Sphären von öffentlich und privat oder von Ökonomie und Intimität zu einer winevitable contamination and disorder« (Zelizer 2005, 21). Dabei sind ökonomische Transaktionen und intime Verbindungen in alltäglichen Praktiken - ob im Privathaushalt, der durch intensive ökonomische Interaktionen der Mitglieder gekennzeichnet ist oder im Bereich der Erwerbsarbeit, wo enge persönliche Beziehungen gepflegt werden - auf vielfältige Art und Weise miteinander verstrickt (Zelizer 2005, 33 ff.). ${ }^{8}$ Für die Handlungspraktiken der Akteur_innen werden hegemoniale gesellschaftliche Wert- und Moralvorstellung bedeutend, um »complicated stories and practices« (Zelizer 2005, 12) für Situationen $\mathrm{zu}$ konstruieren, in denen sich ökonomische Transaktionen mit Intimität vermischen. In der Rekonstruktion des Interviewmaterials zeigt sich deshalb - so meine Argumentation - eine affektive Dekommodifizierung (Smietana 2017). Um der Unvereinbarkeit von Geld und Liebe in der Elternwerdung entgegenzuwirken, muss die Beziehungstransformation zwischen den Tragemüttern und werdenden Eltern betont werden. Durch die Etablierung einer persönlichen, triadischen Beziehung zwischen der Tragemutter und den Eltern, so Bleisch (2012), wird die Tragemutter von den Eltern als Person geschätzt und nicht aufgrund der Tatsache, dass sie ein Kind für die beiden austrägt. Das Verhältnis zwischen den Dreien ist demnach nicht als ein Arbeits- oder Dienstleistungsverhältnis zu verstehen, das mit der Geburt endet, sondern als eine persönliche triadische Beziehung, die von Dauer ist und darüber hinaus anerkennt, dass nicht nur zwei, sondern (mindestens) drei Parteien "in einer moralisch bedeutsamen Weise« (Bleisch 2012, 17) an dem Kind und der Familiengründung beteiligt sind. So sind die Eltern und die Tragemutter nicht ausschließlich Vertragspartner_innen, sondern schätzen und achten sich als Personen. ${ }^{9}$

8 Zu den zentralen Vorreiterinnen in dieser Debatte gehören Silvia Federici (2012) zur Diskussion um Lohn für Hausarbeit sowie Regina Becker-Schmidt (1987) zur feministischen Kritik der Trennung von Reproduktions- und Produktionssphäre. Auch andere verweisen darauf, dass Frauen und Kinder im Kontext von Familie Marktmechanismen ausgeliefert sind (Olsen 1983) und die Ehe für Frauen ein Ort der ökonomischen Austauschprozesse und repressiver Machtverhältnisse sein kann (Okin 1989).

9 Auch Rudrappa $(2015,174)$ argumentiert, dass die persönliche Beziehung zwischen den intendierten Eltern und der Tragemutter die Tragemutterschaft ethisch vertretbarer mache. 
In Anlehnung an Bleisch werde ich das Bild einer Triade übernehmen, um mich auf die Reproduktionsbeziehung zwischen intendierten Eltern und Tragemutter zu beziehen, die es ohne die geplante vierte Person, dem Kind, nicht gäbe.

Im nächsten Abschnitt analysiere ich, wie die Paare diese Transformation in Geschichten rund um die Schwangerschaft zum Ausdruck bringen. Im Zuge dieser Transformation haben Anekdoten und episodische Erzählungen eine besondere Bedeutung, weil in ihnen intime Erfahrungen, Emotionen, Vertrauen und Commitment in der Triade von intendierten Eltern und Tragemutter verhandelt werden. Dabei konzentriere ich mich auf die Bedeutung von Schwangerschaftstests als konstitutives Element der Beziehungswerdung.

\section{"Get ready for the longest two minutes" - Schwangerschaftstests als Beziehungskonstitution}

Während der Schwangerschaft hat die Tragemutter die zentrale Aufgabe, die Schwangerschaft für die werdenden Väter zu übersetzen und diese in den Prozess und Verlauf der Schwangerschaft zu integrieren. Die väterliche Subjektposition ist in Schwangerschaften ohnehin eher "fragil", weil sie von der Vermittelbarkeit der kinästhetischen Erfahrungen der Schwangeren abhängig ist (Sänger et al. 2013, 65). Um diesen Erlebensvorsprung zu minimieren und die Väter zu sogenannten »Ko-Schwangeren (Hirschauer et al. 2014) zu machen, schickt die Tragemutter den Vätern z. B. Fotos ihres schwangeren Bauches. Die Väter versuchen ihrerseits einen aktiven Beitrag zu leisten, indem sie an wichtigen Ultraschalluntersuchungen persönlich oder per Skype teilnehmen oder emotionalen Rückhalt geben, wenn die Tragemutter Unterstützung braucht.

Eine besondere Bedeutung für die Beziehungswerdungen haben Anekdoten über gemeinsame Erlebnisse, die von den Paaren retrospektiv als Regelbruch oder als ungewöhnlich gerahmt werden. Dazu gehört u.a. das Missachten der von der Agentur und/oder Fertilitätsklinik aufgestellten Regeln. Diese gemeinsamen widerständigen Praktiken führen dazu, dass sich das Paar und die Tragemutter als eigenständige Beziehungstriade konstituieren können. Sie sind zentral für die Herstellung von Intimität, weil sie auf Vertrauen, geteilten Erfahrungen und Geheimnissen beruhen oder körperliche Informationen, Verletzbarkeiten und peinliche Situationen miteinander geteilt werden (Zelizer 2011, 275 f.). Kurz: Die Triade (er)findet zur Verstetigung ihrer Beziehung gemeinsame soziale Prakti- 
ken, die direkt mit dem zu erfüllenden Auftrag (der Schwangerschaft) zusammenhängen und gleichzeitig ihre affektive Verbundenheit und Intimität bestärken.

In diesem Kontext sind Geschichten zu Schwangerschaftstests ein konstitutives Element der Beziehungswerdung. So erzählen einige der Paare, dass der erste Test gegen den Ratschlag der behandelnden Ärzt_innen alleine von den Tragemüttern zuhause und vor Ablauf der vorgesehenen zehn Tage bis zum Bluttest durchgeführt wurde. Auch Ingo erzählt, dass sie am Tag des Embryotransfers einen Schwangerschaftstest für Despoina gekauft haben, obwohl Agentur und Klinik darauf hingewiesen haben, bis zum offiziellen Bluttest durch die Klinik zu warten. "Ja dann haben wir noch einen Schwangerschaftstest gekauft. Das weiß ich noch, weil da war ihr Mann noch zuhause. Das war dann irgendwie so lustig, da haben wir immer so rumgejoket so, dass es wohl auch die ersten Männer sind, die ihrer Frau einen Schwangerschaftstest kaufen (lacht auf)« (Int. 1, Paar Droste).

Die Situation scheint lustig, weil hier eine soziale Norm nicht erfüllt wird, die sowohl Ingo und Dieter als auch Despoina und ihr Mann teilen: Es ist ungewöhnlich, dass zwei Männer einen Schwangerschaftstest kaufen, während die Erwartung - das suggeriert Ingos Lachen - zu sein scheint, dass Frauen bzw. werdende Mütter für Schwangerschaftsbelange verantwortlich sind. Im Fall von Ingo, Dieter und Despoina wird es aber aufgrund des Tragemutterschaftsarrangements keine (soziale) Mutter geben. Also übernehmen die beiden Männer als werdende Väter und Eltern mit dem Kauf des Schwangerschaftstests die Verantwortung für die Hervorbringung der Schwangerschaft. Sie knüpfen an bekannte und anerkannte soziale Praktiken im Kontext von Schwangerschaft an und machen sich die Schwangerschaft zu eigen. Tests sind laut Hirschauer et al. $(2014,45)$ ein "wichtiges Artefakt im Prozess des 'Schwangergehens` und der Herstellung der Schwangerschaft als Tatsache«. Durch den Kauf des Schwangerschaftstests nehmen Ingo und Dieter aktiv an der Schwangerschaft teil. Zum einen entlasten sie Despoina von Aufgaben, die sonst der werdenden Mutter zukommen würden, zum anderen entkoppeln sie Mutterschaft von Schwangerschaft und damit Despoina symbolisch von dieser Rolle. Durch diese Dezentrierung bekräftigen sie gleich zu Beginn der Schwangerschaft ihre Alleinstellung als werdende Eltern.

Darüber hinaus widersetzen sie sich der medizinischen Kontrolle und schaffen sich ihre eigenen intimen Räume, weil Despoina den Schwangerschaftstest noch vor ihrer ersten offiziellen Untersuchung in der Klinik macht. Sie versuchen sich situativ unabhängig von den Vorgaben derjeni- 
gen Akteur_innen zu machen, die der ökonomischen Sphäre zugeordnet werden.

Während Ingo und Dieter beleuchten, wie sie als werdende Eltern die Schwangerschaft hervorbringen, zeigt die Erzählung von Gavriel und Leshem wie auch die Tragemütter die Schwangerschaft als gemeinsame Erfahrung der Triade inszenieren. Die beiden sind zum Zeitpunkt des ersten Interviews noch am Beginn der Schwangerschaft. Leshem erzählt, dass Tragemutter Gita extra einen digitalen Test kauft, der das Ergebnis vereinfacht als Yes oder No anzeigt. Sie inszeniert die sSchwangerwerdung< mit einem Handyvideo, das sie an die beiden per WhatsApp mit den Worten "Get ready for the longest two minutes" verschickt. "She told us ^Don't forward. Watch the whole two minutes" ", erklärt Gavriel lachend. Sie müssen - auf Gitas Regieanweisung hin - das Video zwei Minuten lang von Anfang bis Ende schauen. Der Prozess des Wartens ist wesentlicher Teil der Inszenierung, die für den Aufbau eines gegenseitigen Vertrauens und das gemeinsame Einstimmen auf die Schwangerschaft wichtig ist. Gita übernimmt die Regie: Sie antizipiert die Vorfreude des Paars und verleiht der Schwangerwerdung eine der Situation angemessene Dramatik. Sie hat eine machtvolle eigenständige Rolle in der Beziehung, da sie den beiden werdenden Vätern die frohe Botschaft über den positiven Test verkündet und nicht etwa eine Ärztin.

Die beiden Episoden verweisen auf die Relevanz von wechselseitigem Vertrauen und Nähe, auf die Bedeutung des Teilens von Geheimnissen, von intimem und privatem Körperwissen ${ }^{10}$ sowie auf die besondere Gemeinsamkeit in diesen Beziehungen. Diesen Handlungen wird von den befragten Paaren eine besondere Bedeutung beigemessen, nicht nur, um ihre persönliche Involviertheit als werdende Eltern zu inszenieren, sondern auch um zu betonen, dass die Beziehung mehr ist als ein vertragsbasierter ökonomischer Tausch. Die Beziehung ist insofern besonders, als dass die Tragemutter z.B. intimes und privates Wissen über ihren Körper mit den Vätern teilt und sie zu Dritt gemeinsam - im Sinne von kooperativ die Schwangerschaft und die zukünftige Familienwerdung hervorbringen. Gleichzeitig greifen sie auf bekannte Praktiken rund um die Hervorbringung einer Schwangerschaft zurück. Sie halten sich an (hetero)normative Vorgaben, wie sie für die Gestaltung von Nahbeziehungen und intime Praktiken im Bereich von Reproduktionsentscheidungen und Familien-

10 Nach Keller und Meuser (2011, 9) geht Körperwissen aus Sozialisationsprozessen und alltagsweltlichen Erfahrungen hervor und beschreibt u.a. "das private und intime Wissen«, das Individuen über den eigenen Körper haben. 
planung vorgesehen sind (Liebsch 2014, 32 f.). Hier wird die Relevanz ideologischer und moralischer Strukturen für die Rahmung ihrer Geschichten sowie ihr Bemühen um Authentizität und Glaubhaftigkeit deutlich.

\section{Solidarität als Gleichheitsnarrativ in der Familienwerdung}

Ein weiteres Narrativ in der Beziehungswerdung ist das der Gleichheit oder der »Augenhöhe«. Wie schon Zelizer (2005; 2011) deutlich macht, involvieren intime Beziehungen den Austausch von Geld. Da Intimität in einem sozialen Kontext relational aufeinander bezogener Akteur_innen hervorbracht wird, ist sie immer auch durch Geschlecht, ökonomisches und kulturelles Kapital, rassifizierte Zuschreibungen sowie Sexualitäten strukturiert und dadurch vermachtet (Boris / Parreñas 2010). Dieser Aspekt wird aber in den Erzählungen ummantelt. So stellen Anton und Frank, die zum Interviewzeitpunkt bereits Väter eines wenige Monate alten Sohnes waren, den solidarischen Aspekt des gemeinsam Er- und Durchlebten in den Vordergrund, um das Spannungsverhältnis zwischen Ökonomie und Intimität zu lockern. Nachdem sie mir in aller Ausführlichkeit über den Prozess der Tragemutterschaft berichtet haben, frage ich, wie sie ihre Beziehung zu der Tragemutter Anna beschreiben würden. Die Antwort bedarf eines vorsichtigen Abwägens und Herantastens an eine als zufriedenstellend empfundene Darstellung:

Anton: [...] Es ist vielleicht jetzt nicht in dem Sinne, dass wir jetzt Teil der Familie sind, das nicht. Aber es ist eine besondere Gemeinsamkeit die man hat.

Frank: Genau. Besondere Dinge kann man manchmal nicht so gut übersetzen. Das ist eine Art von einer Gemeinsamkeit, die kann man nicht gut mit Familie im herkömmlichen Sinne übersetzen, weil es eben eine andere Form von Familie ist, wenn man unbedingt den Begriff verwenden will.

Anton: Und auch nicht unbedingt eine Freundschaft. Es ist so eine Art, also ich finde empfinde das immer so ein bisschen wie so eine Solidarität zwischen Familien oder so etwas.

Frank: Also sie hat uns geholfen eine Familie zu werden und so glaube ich, nimmt sie es wahr.

$[\ldots]$ 
Anton: Also ich mein' sie wird irgendwelche Raten von ihrer Hypothek auf dem Haus abzahlen [...] Also das hört sich jetzt so'n bisschen komisch an, aber letztendlich also -

Frank: Also auf eine gewisse Weise helfen wir ihr dabei. Das Haus ist natürlich jetzt nicht die Familie, aber irgendwie hängt es so ein bisschen miteinander zusammen. (Int. 1, Paar Arnold)

Ihre Reflexion über die Beziehung zeigt zunächst, dass ihnen das Vokabular fehlt, die Komplexität ihrer Beziehung zu Anna angemessen zu beschreiben. Wie Frank erklärt, ist es keine Familie im »herkömmlichen Sinne«. Anton verweist aber auf die »besondere Gemeinsamkeit«, die sie mit Anna teilen. Aber was zeichnet diese Gemeinsamkeit aus? Die Gemeinsamkeit, so meine Deutung, liegt in der geteilten Erfahrung der Tragemutterschaft und sie begründet ihr intimes Verhältnis: Über lange Zeit hat Anna mit Anton und Frank ihr kinästhetisches Erleben sowie (medizinisches) Wissen über sich und ihren Körper geteilt. Anton und Frank mussten Anna in vielen Belangen vertrauen, waren von ihrer Unterstützung und Kooperation abhängig. Und auch nach der Geburt des Kindes tauschen sie sich regelmäBig über ihre Familien aus, schicken sich Fotos und Emails.

Für Anton ist die Beziehung keine Freundschaft, vielleicht weil diese aus seiner alltagsweltlichen Logik heraus einer anderen Komposition z. B. hinsichtlich der Entstehungsgeschichte und geteilter Interessen bedürfte. Durch die Adressierung von Solidarität bringt er aber etwas stark Gemeinschaftliches zum Ausdruck. Solidarität beruht auf der Annahme geteilter Werte wie der Bedeutung von Familie und auf gegenseitiger Unterstützung. Man steht füreinander ein und fühlt sich gemeinsam einer Sache gegenüber verpflichtet. In dieser Suchbewegung nach den richtigen Begrifflichkeiten wird deutlich, dass Anna in dieser Erzählung nicht Teil ihrer Kern- oder Zweielternfamilie ist, aber sie hat ihnen "geholfen eine Familie zu werden«. Sie hat das Paar zu einer Familie gemacht. Dadurch wird sie wieder in die Familie hereingeholt und die Ambivalenz in den intimen Verstrickungen zwischen Anna und dem Paar kommen zum Ausdruck. Familie wird im Kontext von Tragemutterschaft immer durch weitere Personen (wie die Tragemutter, die Eizellproduzentin, das medizinische Personal, etc.) mithervorgebracht, die aber nicht zur Familie bzw. nicht zum Kern der Familie gehören. Um Anna sprachlich einzubinden, bemüht Anton den Begriff der Solidarität, der die affektive Verbundenheit mit ihr hervorheben soll. Als Helfende hat Anna eine machtvolle Position. Sie hat Anton und Frank dazu verholfen, etwas zu werden, was sie ohne sie nicht wären: eine Familie mit Kind. 
Somit dient die Solidarität auch als Gegennarrativ zum Marktverhältnis, weil sie einen Austausch unter Gleichen suggeriert, der sozialen Zusammenhalt schafft (Bourdieu 1998, 170). Die Deutung der Beziehung als eine solidarische kann als Kunstgriff interpretiert werden, der verdeckt, dass es sich um einen ungleichen Tausch handelt und ihr solidarisches Handeln nur bedingt reziprok und symmetrisch ist. Die in ihrer Beziehung angelegten Widersprüche spiegeln Radins (1996) Konzept der incomplete commodification wider: Es findet eine Inwertsetzung von Schwangerschaft und Geburt statt, aber eben nicht vollständig, weil sie auf der Beziehungsebene deutlich abgemildert wird. Anton spricht die ökonomische Dimension in ihrer Beziehung aus, verweist aber gleichzeitig auf die Strukturähnlichkeit der gegenseitigen Unterstützung. Während Anna ihnen hilft, eine Familie zu werden, unterstützen sie Anna dabei, eine Hypothek des Hauses ihrer Familie abzubezahlen. Sie haben unterschiedliche Bedürfnisse, die zwar mit anderen Mitteln befriedigt werden, aber - und das zeichnet für ihn wiederrum die Gleichwertigkeit aus - sie helfen sich gegenseitig.

Der Solidaritätsbegriff ist mit Blick auf die Anerkennung der reproduktiven Tätigkeiten mit Ambivalenz behaftet: Anton und Frank werten Annas reproduktive Leistungen auf und machen diese als Arbeit sichtbar, weil sie dafür etwas für die Absicherung ihrer eigenen Familie bekommt. Gleichzeitig verdeckt das Solidaritätsnarrativ die Asymmetrie in der wechselseitigen Unterstützung und den Umstand, dass die Reziprozität in ihrer Beziehung begrenzt ist. Die Arbeit geschieht hier genauso wenig wie in anderen Intimbeziehungen (z. B. männliche Alleinernährerfamilie) (ausschließlich) aus Liebe. Insofern hat Anton recht, wenn er kommentiert, "das hört sich jetzt so ein bisschen komisch an". Denn schlussendlich legt er damit offen, dass Geld in intimen Beziehungen mit Unbehagen besetzt ist. Gerade deshalb ist es wichtig, dass Frank betont, dass sie Anna "auf eine gewisse Weise" auch helfen. Zwar ist die Hilfe nicht vergleichbar, denn "das Haus ist natürlich nicht die Familie«, aber - und darauf kommt es an - es hängt "irgendwie [...] so ein bisschen miteinander zusammen." In dieser Logik ist es auch Hilfe. Das "irgendwie " hat eine Entlastungsfunktion. Frank kann sich darauf zurückziehen, nicht genau zu wissen, wie die beiden Hilfen miteinander zusammenhängen. So bleibt gesichert, dass sie eine Beziehung mit Anna führen, die auf Gegenseitigkeit und Gleichheit beruht. Anna wird zugesprochen, dass sie etwas Besonderes für die beiden getan hat und sie bekommt für ihre reproduktive Arbeit Bestätigung und Anerkennung. Gleichzeitig müssen Anton und Frank die Asymmetrie in der Beziehung abmildern, indem sie betonen, dass sie als Triade eine »besondere Gemeinsamkeit» teilen, die sich in dem gemein- 
samen durchlebten Prozess der Tragemutterschaft, Schwangerschaft und Geburt materialisiert.

\section{Fazit und Ausblick: Ambivalenzen zwischen Markt und Intimität}

Mit der Rekonstruktion des Interviewmaterials habe ich gezeigt, wie die Paare in der Beziehungswerdung zur Tragemutter die ökonomischen Aspekte verdecken. Gemeinsam mit der Tragemutter (er)finden sie soziale Praktiken, um Intimität in der zunächst zweckgebundenen und kommerzialisierten Beziehung herzustellen und zu stärken. Dies ist wichtig, um sich als Triade unabhängig von der Agentur zu konstituieren und um den Widerspruch zwischen Geld und Intimität zu "zähmen" (Wimbauer 2003).

In ihren Erzählungen darüber, wie sie sich kennenlernten und in den Anekdoten zu den Schwangerschaftstests geben sie ihrem Handeln eine moralische Rahmung und orientieren sich dabei an alltagsweltlich akzeptierten Praktiken. So eignen sie sich bekannte Praktiken aus dem Feld der Schwangerschaft an, um gemeinsam die Schwangerschaft hervorzubringen. Dabei werden die Paare als die werdenden Eltern adressiert und durch entsprechende Inszenierungen der Tragemutter von ihr unterstützt, Eltern $z u$ werden. In dieser Resignifikation liegt ambivalent subversives Potential (Butler 1991): Heterosexuelle reproduktive Praktiken werden zunächst irritiert. Die Paare werden zu anerkannten Ko-Schwangeren gemacht, während die Schwangerschaft zum einen von der schwangeren Person und zum anderen von Mütterlichkeit dezentriert wird (Hirschauer et al. 2014). Gleichzeitig wird die heterosexuelle Norm durch den Gebrauch von Reproduktionstechnologien z. B. dadurch verstärkt, dass sich die Väter eine biologische Verwandtschaft zu dem Kind wünschen, damit die Norm der Zweielternfamilie erhalten bleibt. Das sorgfältige boundary management der Paare, durch welches sie die Tragemutter von ihrer Familie als Elternpersonal dezentrieren, hat auch die Funktion, dem Normalitätsanspruch der Zweielternfamilie gerecht werden können.

Doch die Tragemutter fehlt eben nicht in ihren Erzählungen. Zum Teil konstruieren die Paare Familienbilder außerhalb heteronormativer Vorstellung der traditionellen Kleinfamilie, weil sie die Tragemutter zu einem selbstverständlichen Teil ihrer Familienwerdungsgeschichte machen auch wenn ihnen für die Bezeichnung dieser Beziehungen (noch) die passenden Begrifflichkeiten fehlen. Doch die reproduktiven Praktiken sind 
stark stratifiziert und spiegeln sozioökonomische Ungleichheiten wieder: Wer Zugang zu diesen Technologien hat, und wer die reproduktive Arbeit gegen Geld verrichtet, hängt von den ökonomischen Ressourcen, der rechtlichen Anerkennung sowie von Geschlecht und Sexualität ab.

Zusammenfassend zeigen die Rekonstruktionen aus meinem eigenen Material sowie die Ergebnisse anderer Studien, dass die Konstruktion von affektiven Beziehungen bei gleichzeitiger Verschleierung der Kommodifizierung reproduktiver Praktiken bedeutend ist, um ein biologisch-genetisches Normalitätsideal herzustellen. Die Abweichung davon kann von den Väterfamilien (anders als von heterosexuellen Paaren) nicht vertuscht werden. Zudem werden sie als Beziehungs- und Elternkonstellation aufgrund heteronormativer Familienvorstellungen von dem gesellschaftlichen AuBen als nicht verantwortungsvolle Eltern gelesen. Diese grounded everyday moralities (Plummer 2003) müssen sie im Vorhinein antizipieren und sich reflexiv in ihrem reproduktiven Handeln auf sie beziehen.

\section{Literatur}

Andrews, Lori B. (1995): Beyond Doctrinal Boundaries: A Legal Framework for Surrogate Motherhood. In: Virginia Law Review 81 (8), 2343-2375.

Boris, Eileen / Parreñas, Rhacel S. (Hrsg.) (2010): Intimate Labors. Cultures, Technologies, and the Politics of Care. Stanford.

Becker-Schmidt, Regina (1987): Die doppelte Vergesellschaftung - die doppelte Unterdrückung: Besonderheiten der Frauenforschung in den Sozialwissenschaften. In: Unterkircher, Lilo / Wagner, Ina (Hrsg.): Die andere Hälfte der Gesellschaft. Wien, 10-25.

Bleisch, Barbara (2012): Leihmutterschaft als persönliche Beziehung. In: Jahrbuch für Wissenschaft und Ethik 17 (1), 5-27.

Bourdieu, Pierre (1998): Praktische Vernunft. Zur Theorie des Handelns. Frankfurt a. M.

Butler, Judith (1991): Das Unbehagen der Geschlechter. Frankfurt a. M.

Colen, Shellee (1995): Like a mother to them: Stratified reproduction and West Indian childcare workers and employers in New York. In: Ginsburg, Faye/Rapp, Rayna (Hrsg.): Conceiving the New World Order: The Global Politics of Reproduction. Berkeley, $78-102$.

Cooper, Melinda / Waldby, Catherine (2014): Clinical Labor. Tissue Donors and Research Subjects in the Global Bioeconomy. Durham/London.

Corbin, Julie/Strauss, Anselm (2008): Basics of Qualitative Research: Techniques and Procedures for Developing Grounded Theory. Thousand Oaks.

Corea, Gena (1985): The Mother Machine. Reproductive Technologies from Artificial Insemination to Artificial Wombs. New York.

Deppermann, Arnulf (2013): Interview als Text vs. Interview als Interaktion. In: Forum Qualitative Sozialforschung 14 (3, Art. 13), 61 Absätze. 
Federici, Silvia (2012): Aufstand aus der Küche. Reproduktionsarbeit im globalen Kapitalismus und die unvollendete feministische Revolution. Münster.

Hirschauer, Stefan/Heimerl, Birgit/Hoffmann, Anika/Hofmann, Peter (2014): Soziologie der Schwangerschaft. Exploration pränataler Sozialität. Stuttgart.

Lettow, Susanne (2011): Biophilosophien. Frankfurt/New York.

Liebsch, Katharina (2014): Genetische Intimität. Zur (Neu-)Regulierung enger Beziehungen und körperlicher Verbundenheit durch Gendiagnostik. In: feministische studien 32 (1), 24-38.

Moreno, Adi (2016): Families on the Market Front. In: Hoffman, Susanne/Moreno, Adi (Hrsg.): Intimate Economies. Bodies, Emotions, and Sexualities on the Global Market. New York, 233-259.

Okin, Susan Moller (1989): Justice, Gender, and the Family. New York.

Olsen, Frances E. (1983): The Family and the Market: A Study of Ideology and Legal Reform. In: Harvard Law Review 96 (7), 1497-1578.

Parreñas, Rhacel S. / Thai, Hung C. / Silvey, Rachel (2016): Guest Editors' Introduction: Intimate Industries: Restructuring (Im)Material Labor in Asia. In: positions. asia critique 24 (1), 1-15.

Plummer, Ken (2003): Intimate Citizenship: Private Decisions and Public Dialogues. Seattle / London.

Radin, Margaret Jane (1996): Contested Commodities. Cambridge.

Rudrappa, Sharmila/Collins, Caitlyn (2015): Altruistic Agencies and Compassionate Consumers: Moral Framing of Transnational Surrogacy. In: Gender \& Society 29 (6), 937-959.

Sänger, Eva / Dörr, Annalena/Scheunemann, Judith / Treusch, Patricia (2013): Embodying Schwangerschaft: pränatales Eltern-Werden im Kontext medizinischer Risikodiskurse und Geschlechternormen. In: GENDER 2013 (1), 56-71.

Shultz, Marjorie M. (1990): Reproductive Technology and Intent-Based Parenthood: An Opportunity for Gender Neutrality. In: Wisconsin Law Review 1990 (2), 297-398.

Smietana, Marcin (2017): Affective De-Commodifying, Economic De-Kinning: Surrogates' and Gay Fathers' Narratives in U.S. Surrogacy. In: Sociological Research Online $22(2), 1-13$.

Teman, Elly (2010): Birthing a Mother. The Surrogate Body and the Pregnant Self. Berkeley / Los Angeles / London.

Wimbauer, Christine (2003): Geld und Liebe. Zur symblischen Bedeutungen von Geld in Paarbeziehungen. Frankfurt/New York.

Yaron, Lee (2017): Only Three Same-sex Couples Allowed to Adopt in Israel Since It Became Legal in 2008. In: Haartez, 04.04. 2017. https://www.haaretz.com/israel-news/. premium-only-three-same-sex-couples-have-been-able-to-adopt-in-israel-1.5457101 (20.08.2018).

Zelizer, Viviana (2005): The Purchase of Intimacy. Princeton.

Zelizer, Viviana (2011): Economic Lives. Princeton. 